\title{
Transverse resistivity and Hall effect of $d$-wave superconductors with twin boundaries: Numerical solutions of time-dependent Ginzburg-Landau equations in the presence of thermal noise
}

\author{
Qunqing $\mathrm{Li}^{1,3}$ and Z. D. Wang ${ }^{1,2, *}$ \\ ${ }^{1}$ Department of Physics, University of Hong Kong, Pokfulam Road, Hong Kong, China \\ ${ }^{2}$ Department of Material Science and Engineering, University of Science and Technology of China, Hefei, China \\ ${ }^{3}$ Department of Physics, Tsinghua University, Beijing, China \\ (Received 17 October 2000; revised manuscript received 8 February 2002; published 21 October 2002)
}

\begin{abstract}
Taking into account thermal fluctuations, we solve numerically the time-dependent Ginzburg-Landau equations to study the role of twin boundaries on the transverse resistivity as well as Hall effect for a $d$-wave superconductor. In the presence of an external current parallel to the twin boundary, we observe that the twin boundary (TB) not only behaves as a pinning center, but also induces a negative component of transverse resistivity. A sign reversal may occur for the transverse resistivity and Hall signal, changing from negative to positive as the magnetic field increases. The increase of the strength of the TB can enhance the negative transverse resistivity, but will soon saturate at higher twin-boundary strengths. Only the antisymmetric part of the transverse resistivity is significantly affected by the normal-state off-diagonal conductivity, while the symmetric part may reflect a key role of the TB on the anisotropy.
\end{abstract}

DOI: 10.1103/PhysRevB.66.134518

PACS number(s): 74.20.De, 74.25.Fy, 74.60.Ge

\section{INTRODUCTION}

One interesting but puzzling feature of the vortex motion in high-temperature superconducting cuprates is the sign reversal behavior of the Hall resistivity. ${ }^{1-6}$ The Hall resistivity $\rho_{x y}$ undergoes a sign change from positive to negative just below the transition temperature for low applied magnetic field, while according to conventional models of the vortex dynamics, the Hall effect in the mixed state of superconductors should have the same sign as that in the normal state.

To explain the sign change of the Hall resistivity, a number of mechanisms were proposed, including those based on the "particle-hole" asymmetry in the framework of timedependent Ginzburg-Landau (TDGL) theory ${ }^{7,8}$ and vortex pinning. ${ }^{9,10}$ On the one hand, the TDGL equations are generalized by allowing the relaxation time to be complex to obtain a nonzero Hall conductivity $\sigma_{x y}^{v}$. In the framework of BCS theory, it was indicated that $\sigma_{x y}^{v}$ appears as a result of the electron-hole asymmetry. ${ }^{11,12}$ The application of this theory to high-temperature superconductors (HTSC's) leads to a conclusion that the Hall resistivity is hole like in the underdoped regime and Hall sign reversal occurs only in the overdoped regime. But the experimental results have shown that the sign reversal of the Hall resistivity appears in the underdoped and slightly overdoped regimes, ${ }^{13,14}$ suggesting that such a theory is unable to account for the sign reversal phenomena in the mixed state of HTSC's. On the other hand, the vortex pinning effect on the mixed-state Hall effect in high- $T_{c}$ superconductors in the presence of thermal fluctuations, including the sign reversal and scaling behavior, is still debatable. $3,6,9,10,15-18$

Twin boundaries (TB's) are common pinning defects in some HTSC's like $\mathrm{YBa}_{2} \mathrm{Cu}_{3} \mathrm{O}_{7}$ (YBCO), and their pinning properties have been extensively studied. As an alternative to the hydrodynamic and microscopic approaches, we shall focus on the TB's pinning effect on the Hall effect of a $d$-wave superconductor starting from a time-dependent version of the Ginzburg-Landau equations. This method is intermediate between the hydrodynamic and microscopic approaches, in that the time dependence of the order parameter is explicitly considered, while the effects of the quasiparticles are lumped into an effective conductivity for the "normal fluid." To clarify the TB's role on the transverse resistivity and Hall effect, by simulating the $d$-wave TDGL equations we study the transverse vortex motion with a current parallel to the TB. We find the TB not only behaves as a pinning center, but also induces a negative component of the transverse resistivity. By including the contribution from the normal-state Hall effect, we find that as the magnetic field increases the transverse resistivity and Hall signal may undergoe a sign change from negative to positive. The symmetric and antisymmetric (Hall) parts of the transverse resistivity are addressed separately under different conditions. The influence of the strength of the TB is also studied. With increasing TB strength the negative transverse resistivity is enhanced at first, but saturates quickly.

\section{TIME-DEPENDENT GINZBURG-LANDAU EQUATIONS}

We employ a model of an isotropic two-dimensional Fermi liquid with attractive interaction in the $d$-wave channel and repulsive interaction in the $s$-wave channel. The GL theory for such a superconductor has been presented by Ren, $\mathrm{Xu}$, and Ting ${ }^{19}$ based on Gor'kov equations. The TDGL equations may be expressed as follows: ${ }^{20}$

$$
\begin{aligned}
-\gamma \frac{\partial S}{\partial t}=\frac{\partial f}{\partial S^{*}}= & {\left[\alpha_{s}+\frac{4}{3}\left(|S|^{2}+|D|^{2}\right)+\boldsymbol{\Pi}^{2}\right] S } \\
& +\frac{2}{3} D^{2} S^{*}+\frac{1}{2}\left(\boldsymbol{\Pi}_{x}^{2}-\boldsymbol{\Pi}_{y}^{2}\right) D,
\end{aligned}
$$




$$
\begin{aligned}
-\gamma \frac{\partial D}{\partial t}= & \frac{\partial f}{\partial D^{*}}=\left[\alpha_{d}+\frac{8}{3}|S|^{2}+|D|^{2}+\boldsymbol{\Pi}^{2}\right] D \\
& +\frac{4}{3} S^{2} D^{*}+\left(\boldsymbol{\Pi}_{x}^{2}-\boldsymbol{\Pi}_{y}^{2}\right) S \\
-\hat{\sigma}^{(n)} \frac{\partial \mathbf{A}}{\partial \mathbf{t}}= & \kappa^{2}(\boldsymbol{\nabla} \times \boldsymbol{\nabla} \times \mathbf{A})-\kappa\left(\boldsymbol{\nabla} \times \mathbf{H}_{\mathbf{e}}\right) \\
+ & \left\{S^{*} \boldsymbol{\Pi} S+\frac{1}{2} D^{*} \boldsymbol{\Pi} D+\frac{1}{2}\left[S^{*}\left(\boldsymbol{\Pi}_{x}-\boldsymbol{\Pi}_{y}\right) D\right.\right. \\
& \left.\left.+D^{*}\left(\boldsymbol{\Pi}{ }_{x}-\boldsymbol{\Pi}_{y}\right) S\right]+ \text { H.c. }\right\} .
\end{aligned}
$$

In these equations, the two order parameters $S$ and $D$ are normalized by $\Delta_{0}=\sqrt{4 / 3 \alpha \ln \left(T_{d} / T\right)} \quad$ with $\alpha$ $\approx 7 \zeta(3) / 8\left(\pi T_{c}\right)^{2}$, the space by the coherence length $\xi$, and the vector potential $\mathbf{A}$ by $\Phi_{0} / 2 \pi \xi$ with $\Phi_{0}=h / 2 e$ being the flux quantum, respectively. $f$ is the free energy functional, and $\boldsymbol{\Pi}=i \boldsymbol{\nabla}+\mathbf{A}$. Here $\gamma$ is a dimensionless order-parameter relaxation time. It could be a complex number, $\gamma=\gamma_{1}+i \gamma_{2}$ (we simply take $\gamma_{1}=1$ ). The imaginary part $\gamma_{2}$ may give contributions to the Hall effect. ${ }^{7,8}$ The time $t$ is normalized by $\sigma_{x x}^{(n)} \lambda^{2}$ with $\sigma_{x x}^{(n)}$ the zero-field longitudinal normal-state conductivity and $\lambda$ the magnetic penetration depth. $\hat{\sigma}^{(n)}$ is the normal-state conductivity tensor, which is normalized by the $\sigma_{x x}^{(n)}$ :

$$
\hat{\sigma}^{(n)}=\left(\begin{array}{ll}
1 & \sigma_{x y}^{(n)} \\
-\sigma_{x y}^{(n)} & 1
\end{array}\right) .
$$

The off-diagonal conductivity in the low-field limit is of the form $\sigma_{x y}^{(n)}(H)=\omega_{c} \tau \approx(e \tau / m) H=\sigma_{x y}^{(n)}(0) H$ and thus can be considered to be proportional to the applied magnetic field. The applied external current can be included by coupling the Maxwell equation $\kappa \boldsymbol{\nabla} \times \mathbf{H}_{\mathbf{e}}=\mathbf{J}$ to the above TDGL equation (3). ${ }^{20}$ In the above equations, we have chosen such a gauge in which the electrostatic potential does not appear, so the local electric field is simply given by $\mathbf{E}=-\partial_{t} \mathbf{A}$. The longitudinal and transverse resistivities can be obtained as $\rho_{x x}$ $=E_{x x} / J$ and $\rho_{x y}=E_{x y} / J$, respectively, where $E_{x x}$ and $E_{x y}$ are along and perpendicular to the directions of the applied current. For an applied magnetic field along the $z$ direction, the transverse resistivity can be written as two parts: $\rho_{x y}$ $=\rho_{x y}^{s}+\rho_{x y}^{a}$, where $\rho_{x y}^{s}$ is the symmetric part with $\rho_{x y}^{s}(-H)=\rho_{x y}^{s}(H)$ and $\rho_{x y}^{a}$ is the antisymmetric part with $\rho_{x y}^{a}(-H)=-\rho_{x y}^{a}(H)$.

The TB is incorporated by assuming the properties of the system may change sharply across the boundary. ${ }^{20}$ For example,

$$
\alpha_{s, d}=\alpha_{s_{0}, d_{0}}+V_{s, d} \delta\left(y-y_{0}\right)
$$

describes the variation of $\alpha_{s, d}$ across the TB along the line $y=y_{0}$.
Besides, we also include the effect of thermal noise on the vortex motion, which seems to be a merit of the present numerical simulation. As usual, by taking into account the thermal noise force, the previous GL equations (1) and (2) can be written in a form ${ }^{21}$

$$
-\gamma \frac{\partial \Psi}{\partial t}=\frac{\partial f}{\partial \Psi^{*}}+F_{t h}(\mathbf{r}, t),
$$

where $\Psi$ denotes the order parameters $S$ and $D$, and $F_{t h}(\mathbf{r}, t)$ is a dimensionless white noise random force. Similarly, the thermal noise force should also be put in the right-hand side of Eq. (3), which can be realized by writing the applied external current as $\widetilde{\boldsymbol{J}}=\mathbf{J}+\boldsymbol{\delta} \boldsymbol{J}$ with $\boldsymbol{\delta} \mathbf{J} \propto \mathbf{J} \times F_{t h}$. The thermal noise term can be simulated as ${ }^{22}$

$$
\begin{aligned}
F_{t h}(r, t) & \propto \sqrt{\frac{2 \Delta k_{B} T}{p}} \sum_{j} \delta\left(t-t_{j}\right) \Omega\left(t_{j}\right) \Theta\left(p-q_{j}\right) \\
& =F_{t h 0} \sqrt{\frac{2 \Delta T}{p}} \sum_{j} \delta\left(t-t_{j}\right) \Omega\left(t_{j}\right) \Theta\left(p-q_{j}\right),
\end{aligned}
$$

where $p=\Delta / \tau$ is the probability that the noise term acts on a given vortex with $\tau$ the mean time between two successive noise pulses and $\Delta$ the iteration time step. $T$ is the temperature of the superconductor, which is normalized by the critical temperature $T_{c} . F_{t h 0}$ is a dimensionless factor, denoting the strength of the thermal noise. $\Omega\left(t_{j}\right)$ is a random number chosen from a Gaussian distribution of mean 0 and width 1 . $\Theta(x)$ is the unit step function with $\Theta(x)=1$ for $x<0$, and $q_{j}$ is a random number uniformly distributed between 0 and 1 .

\section{NUMERICAL RESULTS}

In our simulation, we choose $\alpha_{s}=1$ and $\alpha_{d}=-1$, which corresponds to a $d$-wave state. ${ }^{20}$ One magnetic flux is thread in a $10 \xi \times 10 \xi$ unit cell for $\kappa=3$, with the periodic boundary condition being used. The periodic arrays of TB's are parallel to the $x$ axis with a transverse spacing of $L=10 \xi$. We apply a transport current $J$ in the $x$ direction; thus vortices would be pinned at the TB until $J$ is greater than depinning current $J_{c}$.

First we set $\gamma_{2}=0$ for a pure dissipative dynamics to study the transverse electrical field at different values of the normal-state off-diagonal conductivity $\sigma_{x y}^{(n)}$. The result is shown in Fig. 1 where $\sigma_{x y}^{(n)}$ is chosen as (a) $\sigma_{x y}^{(n)}=0.0$, (b) $\sigma_{x y}^{(n)}=0.003$, (c) $\sigma_{x y}^{(n)}=0.015$, (d) $\sigma_{x y}^{(n)}=0.024$, and (e) $\sigma_{x y}^{(n)}$ $=-0.015$ by inverting the applied magnetic field. The applied transport current is equal to $J_{x}=0.16$. From Fig. 1(a) we can see a negative transverse resistivity shows up even when there is no contribution from the normal-state offdiagonal conductivity $\sigma_{x y}^{(n)}$. With the increasing of $\sigma_{x y}^{(n)}$, a positive peak arises and increases obviously in the middle of each peak of $E_{x y}$. The contribution from the normal-state off-diagonal conductivity is finally dominant over the effect of TB when $\sigma_{x y}^{(n)}=0.024$. When the magnetic field is inverted, the contributions from these two parts are all nega- 

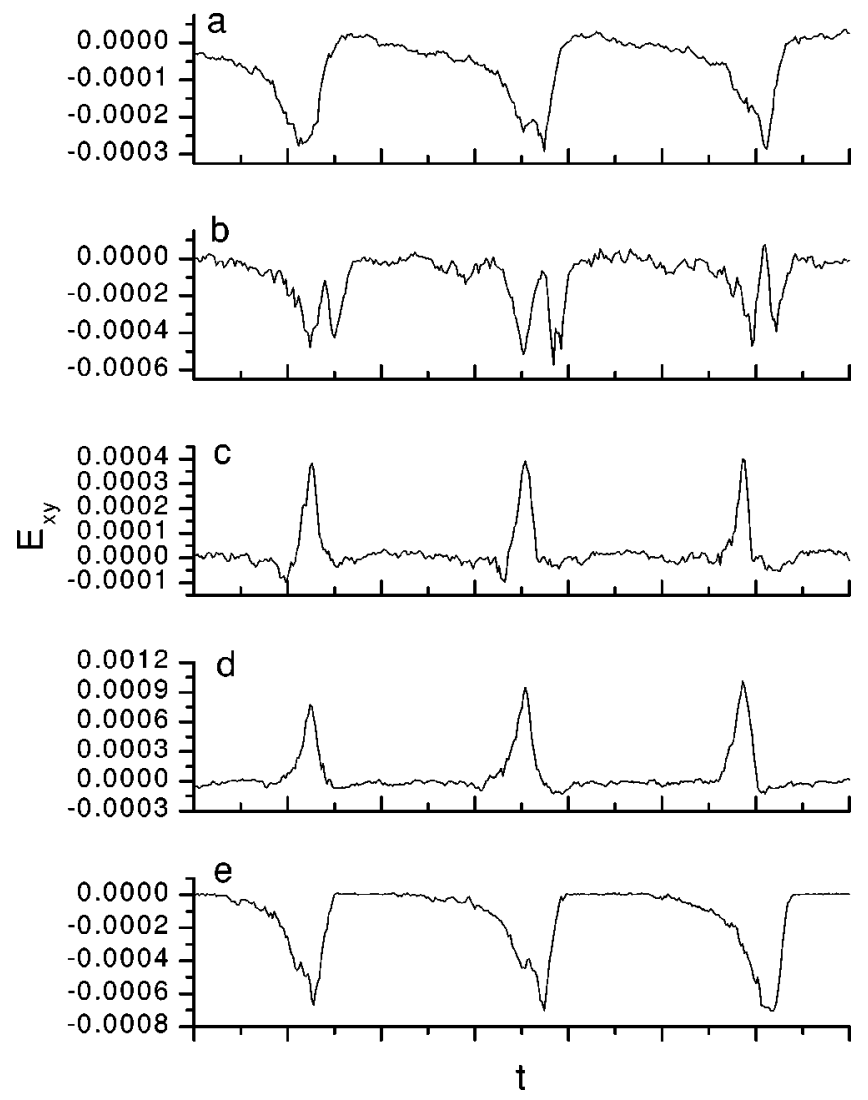

FIG. 1. The time dependence of the longitudinal electrical field $E_{x x}$, the transverse field $E_{x y}$ for $J=0.16$, and $\sigma_{x y}^{n}=$ (a) 0.0 , (b) 0.003 , (c) 0.015 , (d) 0.024 , and (e) -0.015 by inverting the applied magnetic field. Here (and hereafter), the electric fields are in unit of $E_{0}=\Phi_{0} /\left(2 \pi \xi \lambda^{2} \sigma_{x x}^{(n)}\right)$.

tive. Figure 2(a) gives the results of the average transverse resistivity versus $\sigma_{x y}^{(n)}$ under an applied magnetic field and its reversal. Since we adjust the magnetic field by changing the size of the unit cell in our simulation, which would change the density of the TB at the same time, it seems to be a little difficult for us to study the effect of magnetic field on the transverse resistivity directly. But since $\sigma_{x y}^{(n)}$ is proportional to $H$ at low magnetic fields, which is the main effect of the magnetic field on the transverse resistivity, we may consider Fig. 2(a) to illustrate, to some extent, that the transverse resistivity undergoes a sign reversal from negative to positive with increasing the magnetic field at positive low fields. If the applied magnetic field is inverted, the transverse resistivity exhibits a trend which seems symmetric to the curve for the positive field around a horizontal line. In most experiments, the measured Hall resistivity is just the antisymmetric part of the transverse resistivity, $\rho_{x y}^{a}=\frac{1}{2}\left[\rho_{x y}(H)\right.$ $\left.-\rho_{x y}(-H)\right]$. From Fig. 2(b), we can see the Hall resistivity $\rho_{x y}^{a}$ undergoes a sign reversal at low fields and the value of the negative Hall resistivity is relatively small compared to the quickly increased Hall resistivity at higher fields. Moreover, the symmetric part of the transverse resistivity, $\rho_{x y}^{s}$ $=\frac{1}{2}\left[\rho_{x y}(H)+\rho_{x y}(-H)\right]$, is plotted in Fig. 2(c), and it is clear that $\rho_{x y}^{s}$ is almost unchanged at low fields.

Then we turn to change the applied current. In Fig. 3, the
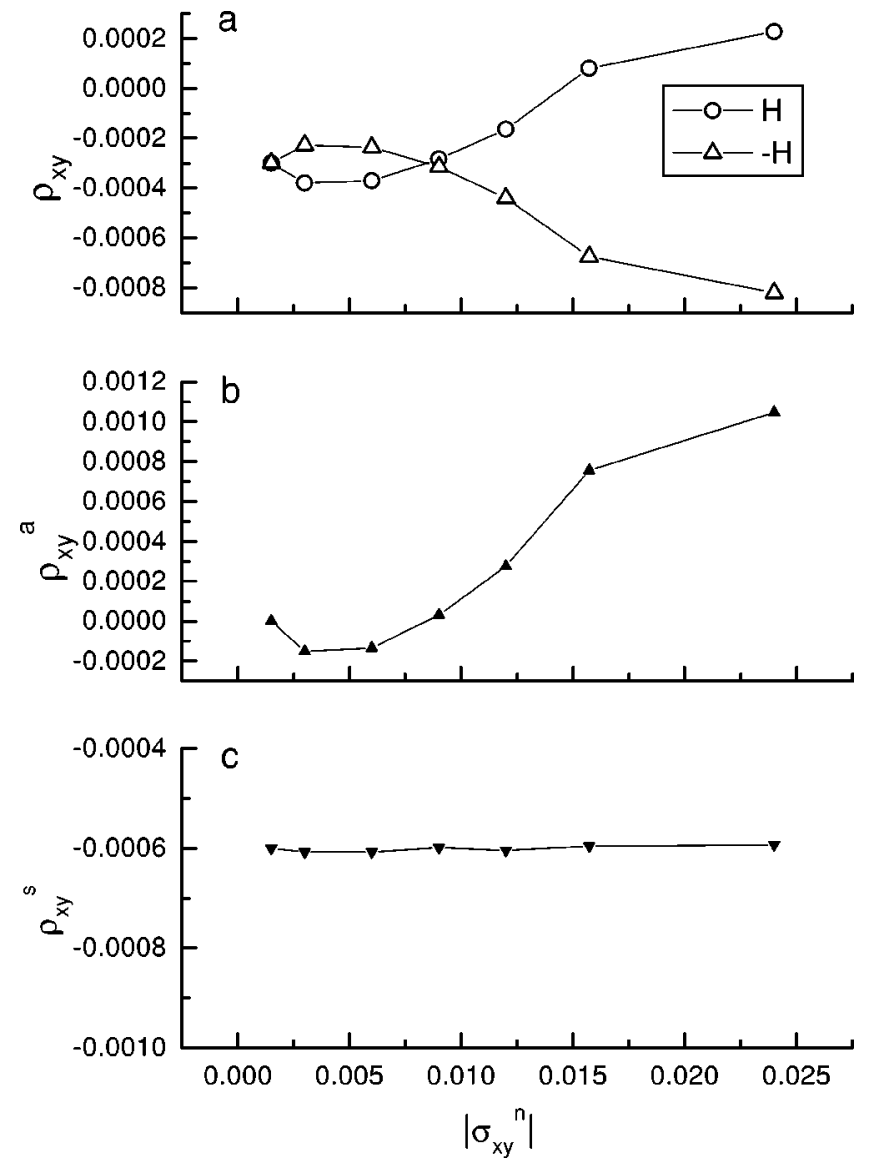

FIG. 2. (a) The average transverse resistivity under a positive and a negative magnetic field, (b) the anti-symmetric part of the average transverse resistivity (Hall resistivity), and (c) the symmetric part of the average transverse resistivity vs the normal-state off-diagonal conductivity. Here (and hereafter), the resistivities are in unit of the inverse normal-state conductivity $\left(\sigma_{x x}^{(n)}\right)^{-1}$.

current $J$ is increased from 0.16 to 0.20 with $\sigma_{x y}^{(n)}=0.003$. Similar to the above case, the transverse resistivity undergoes a sign reversal from negative to positive with an increase of the applied current under a positive magnetic field, while the transverse resistivity is always negative and decreases almost linearly with the applied current when the magnetic field is inverted. The Hall resistivity also undergoes a weak sign reversal at low applied currents and then increases linearly with the applied current, which seems to be qualitatively consistent with experimental results. ${ }^{23}$ It is also interesting that the symmetric part of the transverse resistivity is almost unchanged as well for different applied currents $\left(J>J_{c}\right)$, which seems to imply that $\rho_{x y}^{s}$ is mainly related to the anisotropy of the system in the presence of TB's.

We can adjust the strength of the TB by changing the value of $V_{d}\left(V_{s}\right.$ is irrelevant as long as $\left.\alpha_{s}>0\right)$. Figure 4 gives the influence of the strength of TB's on the transverse resistivity with $J=0.16$ and $\sigma_{x y}^{(n)}=0.003$. Notice here that the magnetic field is kept unchanged, but the applied current is not. Since the depinning current changes for different TB strengths, we choose an appropriate applied current for each 



FIG. 3. Same as Fig. 2 but vs the applied current.

case so that the longitudinal resistivity is unchanged. It can be seen that the transverse resistivity remains negative and decreases with an increase of pinning strength, but saturates soon.

As for thermal fluctuations, we first choose $J=0.14$ with $V_{s}=0.5$ and $V_{d}=1.0$. Vortices are almost pinned by the TB's. Then we increase the strength of thermal fluctuations gradually by adjusting the value of $F_{t h 0}$. In Fig. 5, we set (a) $F_{t h 0}=0.2$, (b) $F_{t h 0}=0.28$, and (c) $F_{t h 0}=0.4$. The thermal energy assists vortices to jump among pinning sites guided by the driving force of the applied current. Each jump corresponds to a peak in both longitudinal and transverse electric fields. It is seen that a positive peak in the transverse field is produced when vortices move (even though we set $\left.\sigma_{x y}^{(n)}=0\right)$. When the value of $\sigma_{x y}^{(n)}$ is greater than zero, only the amplitude of the peak in the transverse field is increased, while the others remain the same. Increasing the strength of the thermal noise enhances the hopping probability of vortices jumping among pinning sites and thus increases both the longitudinal and transverse resistivities.

As a comparison, we also consider the case for a complex relaxation time $\gamma$. Figure 6 presents the results when the imaginary part $\gamma_{2}$ is chosen as $\gamma_{2}=0.01$ and $\gamma_{2}=-0.01$ under an applied current $J=0.16$. The result shows that the Hall resistivity is positive and increases almost linearly with $\left|\sigma_{x y}^{(n)}\right|$ (or the magnetic field) for a negative $\gamma_{2}$, while the Hall resistivity changes from negative to positive and is also linearly dependent on $\left|\sigma_{x y}^{(n)}\right|$ (the magnetic field) for a positive $\gamma_{2}$. This sign reversal in Fig. 6(a) is in agreement with
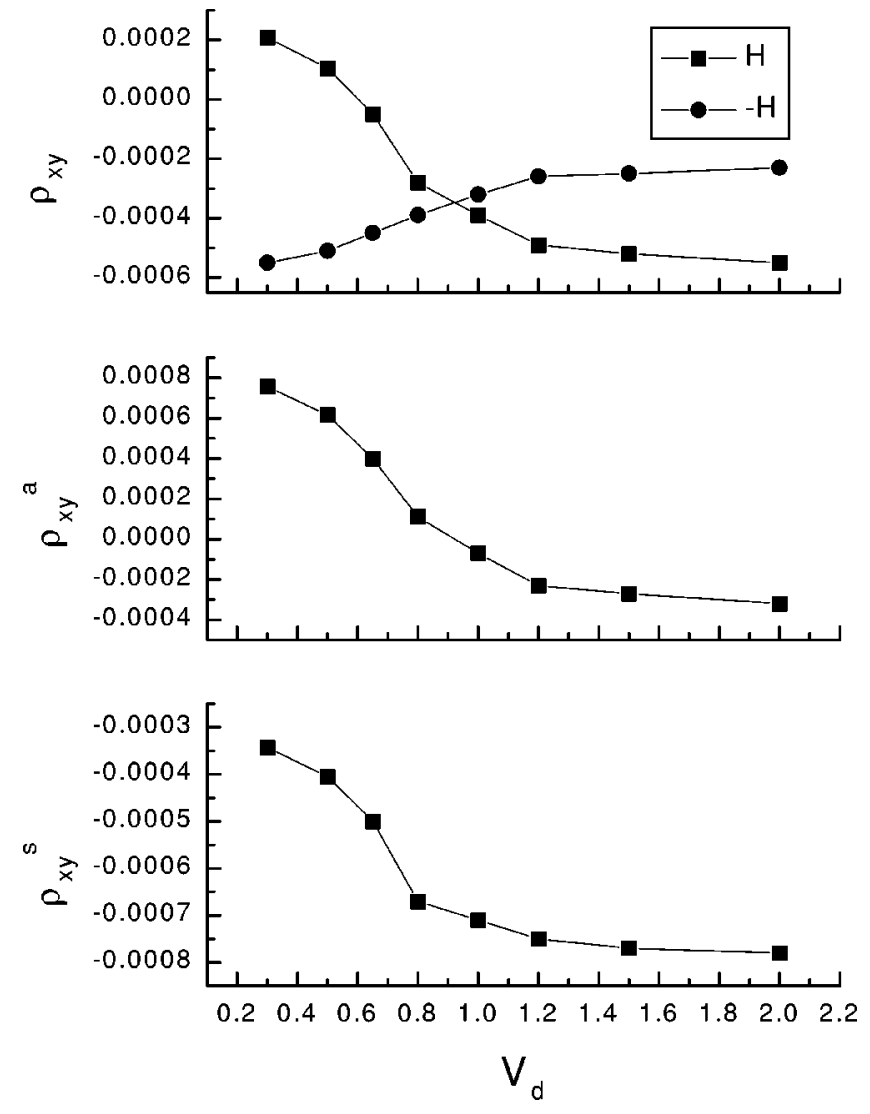

FIG. 4. The transverse resistivity $\rho_{x y}$ and its symmetric and antisymmetric parts vs the TB strength $V_{d}$.

the TDGL analysis of Refs. 7 and 8. The symmetric parts of the transverse resistivity for both cases are almost the same, which are negative and almost unchanged as a function of $\left|\sigma_{x y}^{(n)}\right|$ (the magnetic field), reflecting the anisotropy of the system with TB's.

\section{DISCUSSION}

It is worth pointing out that, in the presence of a nonzero $\sigma_{x y}^{(n)}$ and twin boundaries, neither the particle-hole symmetry nor the $\pi / 2$-rotational symmetry can be expected from the present $d$-wave TDGL equations (1)-(5) even if $\gamma$ is real $\left(\gamma_{2}=0\right) .^{7}$ As a result, the transverse resistivity is neither symmetric nor antisymmetric in the field $H$, and thus the Hall resistivity is nonzero in the cases we consider here (except for the special case $\sigma_{x y}^{(n)}=0$ ), as seen from Figs. 2-5. On the other hand, Fig. 1(a) shows clearly that even when $\gamma_{2}$ and $\sigma_{x y}^{(n)}$ are both zero, a negative transverse electrical field is produced when the vortex begins to move, which is surely due to the TB's. It is known that the TB acts as an easy-flow channel when the Lorentz force is parallel to the TB, but acts as a strong barrier for the force perpendicular to the TB. For the case discussed here, the external applied current is parallel to the TB barrier, so the TB acts mainly as a pinning center. When the applied current exceeds the depinning current, vortices tend to leave the TB with a perpendicular velocity, which increases gradually. But vortices may simulta- 



FIG. 5. The time dependence of $E_{x y}$ for different strengths of the thermal fluctuation $F_{t h 0}$ : (a) 0.2 , (b) 0.28 , and (c) 0.4 .

neously tend to "slide" along the TB direction, thus gaining an increased small velocity parallel to the TB. This parallel velocity contributes the transverse resistivity including both symmetric and antisymmetric (Hall) parts. Looking at Fig. 2, it is natural to ask (i) why the transverse resistivity $\rho_{x y}$ is so small in the negative Hall signal region and (ii) what is the origin of the negative Hall resistivity. In the following, we attempt to give possible answers as well as relevant discussions. (i) In our opinion, some combination of both $\sigma_{x y}^{(n)}$ and twin-boundary strength causes so small $\rho_{x y}$ : a stronger pinning effect of TB's leads to a very small amplitude of $\rho_{x y}$, while $\sigma_{x y}^{(n)}$ intends to increase the mobility of vortices. It is seen clearly from Fig. 2 that $\sigma_{x y}^{(n)}$ affects only the antisymmetric (Hall) component of the transverse resistivity and enhances the positive part after the minimum of Hall component is reached. One can also see from Fig. 4 that both the symmetric and Hall components of the transverse resistivity decrease (and then saturate) when the TB strength increases, with the former being always negative and the latter being from positive to negative; if the strength $\left(V_{d}\right)$ is weaker $(<0.8)$, the Hall component becomes positive while the symmetric component is still negative. (ii) It is interesting to note that the Hall resistivity would be linear in small $\sigma_{x y}^{(n)}$ if there were not the pinning effect due to TB's. It seems that the pinning effect of TB's is mainly responsible for a slightly smaller $\rho_{x y}(H)\left[\right.$ than $\left.\rho_{x y}(-H)\right]$ for very small $\sigma_{x y}^{(n)}$ and thus leads to the small negative Hall signal. ${ }^{24}$

As is known, in most experimental measurements, the antisymmetric part of the transverse resistivity is counted as the
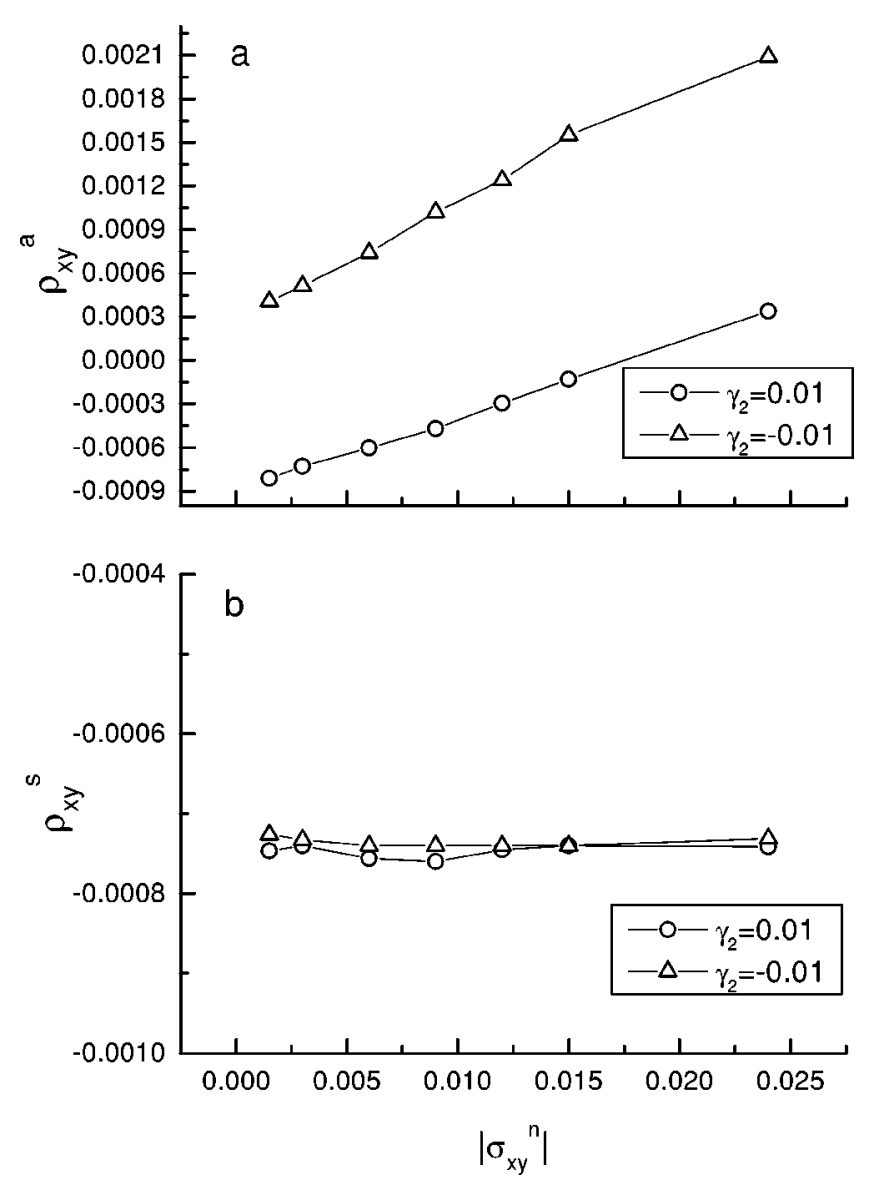

FIG. 6. The antisymmetric part (a) and the symmetric part (b) of the average transverse resistivity with the imaginary part of relaxation time $\gamma_{2}=0.01$ and $\gamma_{2}=-0.01$ vs the normal-state offdiagonal conductivity.

Hall resistivity to avoid spurious effects due to the Hall contact misalignments or due to an inhomogeneous current distribution. The role of the TB on the total transverse resistivity was actually ignored. Just as we show in Fig. 2(b), Fig. 3(b), and Fig. 6(a) for $\rho_{x y}^{a}$, the Hall resistivity is significantly affected by the normal-state off-diagonal conductivity $\sigma_{x y}^{(n)}$, while the symmetric part of the transverse resistivity may give us useful information about the TB's effect, exhibiting the intrinsic anisotropy. This can be seen from the flat curves for $\rho_{x y}^{s}(H)$ in Figs. 2(c), 3(c), and 6(b).

Finally, we wish to indicate that the present work addresses merely low- $\kappa$ cases, where the normal-core approximation, which is quite good for high- $\kappa$ HTSC's, may not be valid. Nevertheless, our results are qualitatively useful for understanding the mixed-state transverse resistivity and Hall effect in $d$-wave HTSC's with TB-like pinning centers.

In summary, taking into account thermal fluctuations, we have solved numerically the $d$-wave TDGL equations to study the role of TB's on the transverse resistivity and Hall effect in the mixed state of HTSC's. In the presence of an applied current parallel to the TB's, we find that TB's not only behave as pinning centers, but also could induce a negative transverse resistivity and Hall signal. With increasing the magnetic field a sign reversal of transverse resistivity and 
Hall signal may occur from negative to positive. Increasing the strength of TB's may enhance the negative transverse resistivity, which is more obvious for low TB strengths. The antisymmetric part of the transverse resistivity is mainly related to the off-diagonal conductivity of the normal state, while the symmetric part may give us more information about the TB's effect on the anisotropy.

\section{ACKNOWLEDGMENTS}

This work was supported by RGC grants of Hong Kong (Nos. HKU 7116/98P, 7144/99P) and Ministry of Science and Technology of China under Grant No. 1999064602. Z.D.W. also acknowledges partial support from the Texas Center for Superconductivity at the University of Houston.

${ }^{11}$ A. G. Aronov, S. Hikami, and A. I. Larkin, Phys. Rev. B 51, 3880 (1995).

${ }^{12}$ A. Van Otterlo, M. Feigel'man, and G. Blatter, Phys. Rev. Lett. 75, 3736 (1995).

${ }^{13}$ T. Nagaoka et al., Phys. Rev. Lett. 80, 3594 (1998).

${ }^{14}$ B. W. Kang et al., Philos. Mag. Lett. 79, 41 (1999).

${ }^{15}$ A. V. Samoilov, Phys. Rev. Lett. 79, 4045 (1997).

${ }^{16}$ L. M. Wang et al., Phys. Rev. Lett. 78, 527 (1997); Phys. Rev. B 59, 14031 (1999).

${ }^{17}$ V. N. Morgoon et al., J. Low Temp. Phys. 105, 963 (1996).

${ }^{18}$ A. Casaca, G. Bonfait, C. Dubourdieu, F. Weiss, and J. P. Senateur, Phys. Rev. B 59, 1538 (1999); A. Casaca, G. Bonfait, M. Getta, M. Lenkens, and G. Muller, ibid. 56, 5677 (1997).

${ }^{19}$ Y. Ren, J. H. Xu, and C. S. Ting, Phys. Rev. Lett. 74, 3680 (1995); J. Phys. Chem. Solids 56, 1749 (1995).

${ }^{20}$ Z. D. Wang and Q. H. Wang, Phys. Rev. B 55, 11756 (1997); Q. H. Wang and Z. D. Wang, ibid. 54, R15 645 (1996).

${ }^{21}$ M. Cyrot, Rep. Prog. Phys. 36, 103 (1973).

${ }^{22}$ A. Brass and H. J. Jensen, Phys. Rev. B 39, 9587 (1989); H. J. Jensen, A. Brass, A. C. Shi, and A. J. Berlinsky, ibid. 41, 6394 (1990); Z. D. Wang et al., ibid. 51, 6119 (1995).

${ }^{23}$ H. C. Yang, L. M. Wang, and H. E. Horng, Phys. Rev. B 56, 99 (1997).

${ }^{24}$ This small negative Hall signal is unlikely induced by some artifacts of the computation precedure as we verified them many times in a variety of cases. But this possibility may not be completely excluded. 